\title{
Erratum: Cubic-quintic nonlinearity in superfluid Bose-Bose mixtures in optical lattices: Heavy solitary waves, barrier-induced criticality, and current-phase relations [Phys. Rev. A 91, 013630 (2015)]
}

Ippei Danshita ${ }^{\circ}$, Daisuke Yamamoto, and Yasuyuki Kato

(Received 4 October 2021; published 13 October 2021)

DOI: 10.1103/PhysRevA.104.049902

Incorrect values were plotted for $w$ and $w_{A B}$ in Figs. 17(a)-17(c) of the original paper due to an error in the numerical calculations. We show the corrected figure below. The correction do not affect any other parts of the original paper.
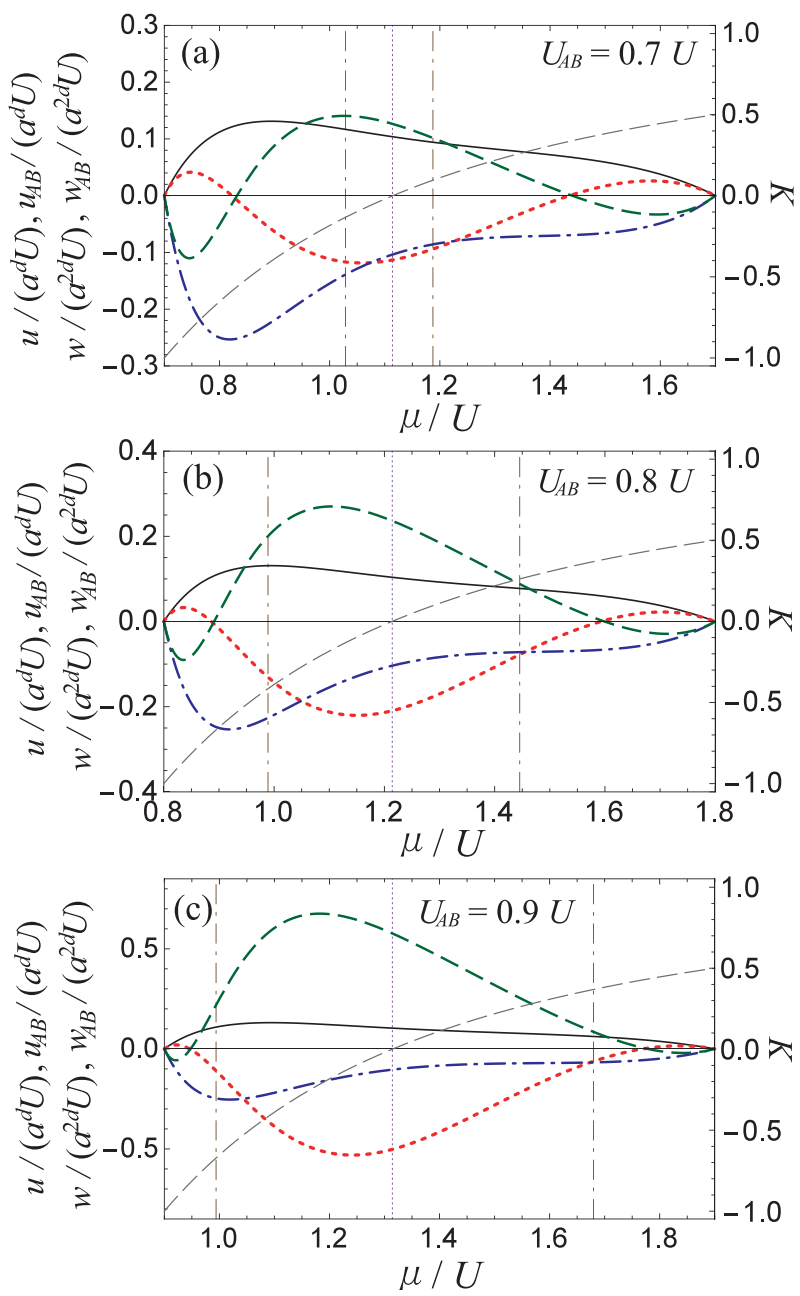

FIG. 17. GL parameters along the metastability limit of the MI state at $v=2$, as functions of $\mu / U$ for $U_{A B} / U=0.7$ (a), 0.8 (b), and 0.9 (c). The solid, thick-dotted, thick-dashed-dotted, thick-dashed, and thin-dashed lines represent $u /\left(a^{d} U\right), u_{A B} /\left(a^{d} U\right), w /\left(a^{2 d} U\right), w_{A B} /\left(a^{2 d} U\right)$, and $K$, respectively. The thin-dashed-dotted and thin-dotted lines mark the TCPs and the $\mu$ value that gives the maximum hopping. 